Published in final edited form as:

Nat Med. 2008 April ; 14(4): 454-458. doi:10.1038/nm1692.

\title{
Detection of colonic dysplasia in vivo using a targeted fluorescent septapeptide and confocal microendoscopy
}

\author{
P. Hsiung, $\mathrm{PhD}^{1}$, J. Hardy, $\mathrm{PhD}^{1}$, S. Friedland, $\mathrm{MD}^{2,3}$, R. Soetikno, $\mathrm{MD}^{2,3}$, C.B. Du, MS ${ }^{1}$, \\ A.P.W. Wu, MD ${ }^{1}$, P. Sahbaie, MD ${ }^{2}$, J.M. Crawford, MD, PhD $^{4}$, A.W. Lowe, MD ${ }^{3}$, C.H. Contag, \\ $\mathbf{P h D}^{1}$, and T.D. Wang, MD, $\mathbf{P h D}^{2,3}$ \\ ${ }^{1}$ Department of Pediatrics, Radiology and Microbiology \& Immunology, Stanford University \\ School of Medicine, Stanford, California \\ ${ }^{2}$ Veterans Affairs Palo Alto Health Care System, Palo Alto, California \\ ${ }^{3}$ Department of Medicine, Stanford University School of Medicine, Stanford, California \\ ${ }^{4}$ Department of Pathology, Immunology and Laboratory Medicine, University of Florida College of \\ Medicine, Gainesville, Florida
}

\begin{abstract}
The merging of targeted probes with new imaging technologies provides a powerful tool for the early detection of cancer. Phage display peptide libraries are highly complex and can be screened for high-affinity ligands with preferential binding to premalignant tissue. An M13 phage library was screened against fresh human colonic adenomas to identify a specific septapeptide sequence, VRPMPLQ, that was synthesized, conjugated with fluorescein, and tested in patients undergoing colonoscopy. Imaging of the topically administered peptide was performed with a novel fluorescence confocal microendoscope delivered through the instrument channel of a standard colonoscope. In vivo imaging at 12 frames-per-second was performed with $50 \mu \mathrm{m}$ working distance with $2.5 \mu \mathrm{m}$ (transverse) and $20 \mu \mathrm{m}$ (axial) resolution. Imaging of the fluoresceinconjugated peptide demonstrated preferential binding to dysplastic colonocytes relative to adjacent normal cells with $81 \%$ sensitivity and $78 \%$ specificity. The methodology described represents a promising diagnostic imaging approach for the early detection of colon cancer and potentially other epithelial malignancies.
\end{abstract}

\section{Keywords \\ in vivo microscopy; molecular imaging; cancer detection; optical fiber confocal}

\section{INTRODUCTION}

Colorectal cancer (CRC) develops in a multistep process that arises from genetic alterations and environmental insults ${ }^{1}$. Most CRCs can be prevented through detection and removal of pre-malignant polypoid lesions ${ }^{2}$. Despite the proven efficacy of endoscopic screening and polyp removal, colorectal cancer remains the third most common cancer in men and women in the United States ${ }^{3}$. The high incidence of colon cancer remains, in part, due to noncompliance to screening guidelines, but also to limitations in conventional endoscopic screening which lacks molecular specificity and reveals only gross anatomic changes through a macroscopic view of the surface mucosa. Flat or depressed neoplasms, which have recently been appreciated as another presentation of colorectal cancer ${ }^{4,5}$, are difficult to detect using endoscopic methods and are associated with an increased risk of submucosal invasion when compared to polypoid lesions of similar size ${ }^{6}$. In addition, patients with a long-standing history of inflammatory bowel disease are at increased risk for developing 
malignancy due to undetected dysplastic lesions ${ }^{7,8}$. These observations support the need for improved methods for detecting early changes in high-risk patients. Novel approaches that are also compatible with conventional endoscopy would allow seamless integration into existing protocols, and offer greater potential for reducing morbidity and mortality. Such an approach could be applicable to other cancers of the epithelium including the esophagus, stomach, cervix, and bladder, which result in $\sim 65 \%$ of all deaths by cancer in the U.S. ${ }^{3}$

A number of biomarkers for CRC have been discovered that may lead to new surveillance methods, identification of high-risk populations, and earlier detection ${ }^{9}$. Because of their high specificity, monoclonal antibodies to these markers have been investigated for tumor detection and drug delivery. Their in vivo use, however, has been limited by immunogenicity and challenges associated with development and production. ${ }^{10}$ In contrast, peptides are typically less immunogenic, non-toxic, and relatively simple to produce in quantity. The small size of peptides may also allow them to penetrate more easily into diseased mucosa, potentially binding to molecular targets at greater tissue depths.

Bacteriophage that display peptides on their surface enables screening of highly diverse libraries for peptides with highly specific binding properties, without prior knowledge of the target. ${ }^{11}$ Phage display experiments have typically been performed using purified receptor targets or established cell lines in culture, leading to selection of phage for those preselected targets enriched on cell lines. Such peptides have been identified through biopanning with human colon cancer cells and have been used as imaging agents in animal models ${ }^{12,13}$. Isolated and cultured cells, however, may de-differentiate and lose tissue-specific traits upon culture ${ }^{14}$. In vitro targeting systems may also fail due to inaccessibility of relevant receptors in vivo ${ }^{15}$. Because phage display enables selection through an iterative process of selection and enrichment, the use of primary premalignant human tissues could eliminate any bias imposed by prolonged culture or over expression of receptors present on end-stage cancer.

Peptides with tissue-specific homing properties have been isolated from animal models in vivo by intravenous injection of native phage libraries and harvesting organs of interest ${ }^{16,17}$. Although ligands and receptors isolated in animal models have been useful for understanding their human homologs, species-specific differences in protein expression and ligand-receptor accessibility may limit their application in humans. For example, TEM7, an endothelial marker corresponding to a transmembrane protein expressed in endothelial cells of human colorectal tumor stroma, is not present in tumor blood vessels but in Purkinje cells in mice ${ }^{18}$. Tissue-homing peptides have also been demonstrated for human tissues, however, recovery of target tissues after intravenous phage administration can only be achieved under special circumstances ${ }^{19,20}$. These approaches have been useful for identifying peptides that bind to abnormal endothelial markers because intravenous phage are confined to the lumen of the blood vessels due to their relatively large size. Selection of peptides through intravenous administration is also biased towards targeting receptors that are directly accessible via the vasculature, and facilitates discovery of molecules more suitable for drug delivery and not for binding to mucosal surfaces that are the object of endoscopic observation. Successful studies using phage display to generate unique probes illustrates a key feature of this method: it is highly selective for the tissues to which the library is exposed. Therefore, if peptides that bind to premalignant human tissues are desired, these tissues should be used in the selection process.

The primary motivation for identifying mucosal markers is to enable topical administration of probes, which maximizes safety and reduces the risk from immunoreactivity-important considerations in the development of molecular imaging approaches. Panning using freshly harvested human tissue has been shown to successfully isolate peptides specifically binding to polarized luminal surfaces of umbilical vein endothelial cells ${ }^{21}$. In addition, peptides specific for endothelial markers for dysplasia have been identified in mice ${ }^{22}$. Previous 
studies with non-specific dyes have shown that confocal microendoscopy is capable of visualizing cellular and subcellular features associated with neoplasia ${ }^{23,24}$. The use of a flexible fibered confocal microscope capable of high speed image acquisition thus may enable subsurface imaging with subcellular resolution, permitting local binding of topically administered dysplasia-targeting reagents to be assessed.

We used an M13 phage library to identify peptides that specifically bind dysplastic colonic mucosa. Nonspecific peptides were first removed with non-malignant intestinal epithelial cells followed by panning with fresh human colonic biopsies. A peptide that binds colonic dysplasia was identified, synthesized, and conjugated with fluorescein for in vivo testing in patients undergoing routine colonoscopy. Fluorescence from topically administered peptide was collected using a confocal microendoscope (Cellvizio®-GI, Mauna Kea Technologies $)^{25-28}$ that can pass through the accessory channel of standard colonoscopes was used to visualize topically administered peptide. Fluorescence images and videos of bound topically administered peptide collected in vivo showed enhanced binding to dysplastic colonocytes compared to the adjacent normal mucosa in the same patient.

\section{RESULTS}

\section{Isolation of phage with specific binding to colonic dysplasia}

Two rounds of incubation with the non-malignant human intestinal cells reduced the original phage library's titer by $97 \%$ to $4.25 \times 10^{9}$ clones. Subsequent incubation of the remaining phage to fresh human colonic tissue further reduced the library to $1.9 \times 10^{5}$ clones after Round 1 of selection. After Round 2 of selection, $7.8 \times 10^{4}$ clones were retained, indicating $\sim 41 \%$ of the clones remained unbound to hyperplastic, inflamed, and focal adenoma. The titer of the eluted phage after the final and third round of panning indicated $\sim 6000$ clones remained after 3 rounds of selection using biopsy tissue.

\section{In vitro assays}

Of the 40 phage clones tested, one clone displaying a peptide with sequence "VRPMPLQ" exhibited 2-3 times increased fluorescence intensity compared to control M13 antibody with no applied phage. Titering of phage binding for this clone indicated approximately 20 times increased binding to HT29 human adenocarcinoma-derived cells relative to HS738 nonmalignant human intestinal cells as compared to wild-type phage lacking a peptide insert, and increased binding was seen compared to 10 other randomly selected clones also eluted from Round 3 of selection. (Fig. 2). Assays performed using fresh biopsies of tubular adenoma from human colon polyps also yielded an $\sim 3$ fold increased fluorescence intensity from abnormal colonocytes lining dysplastic crypts relative to adjacent normal mucosa (Fig. 3). Background fluorescence above the level of autofluorescence was also visible in both polyp and adjacent normal mucosa due to inability to completely remove phage and M13 antibody non-specifically absorbed by the tissue.

Although a number of peptides were identified using this procedure, evaluation of selected peptides in clinical studies necessitated choosing one initial peptide. Since our goal was to identify a peptide reagent capable of targeting dysplasia in a clinically relevant setting, the peptide "VRPMPLQ" was synthesized and conjugated with fluorescein for pilot patient studies conducted during screening colonoscopy.

\section{In Vivo Imaging}

An in vivo image of dysplastic colon compared to adjacent normal mucosa is shown in Figure 4. Fluorescence was correlated to areas containing dysplastic colonocytes as determined by subsequent pathological examination. In contrast, normal mucosa sprayed 
with peptide exhibited generally decreased fluorescence associated with residual peptide collecting in regions between crypts. Peptide also collected in lumens of normal crypts and appeared as round or oval spots of increased fluorescence in the center of a low fluorescence colonocyte layer. Contrast ratio between adenomatous and normal colonocytes was $17.0 \pm$ 4.0 (s.e.m.) with an average signal-to-noise ratio (SNR) of $8.8 \pm 1.0$ (s.e.m.) for $\mathrm{n}=19$ polyps diagnosed as either tubular adenoma or tubulovillous adenoma. The contrast ranged from $0.8 \pm 0.1$ (s.d) (indicating no preferential binding) to $52.3 \pm 6.8$ (s.d) indicating substantial peptide binding.

Figure 5 shows an image taken at the border between a dysplastic polyp and normal mucosa after peptide administration. Imaging of this border region provided a reference point which controlled for variables such as incubation time over a local region of the mucosa and probe contact pressure, which contributes to imaging depth. Contrast between polyp and normal colonocytes calculated using regions within individual images at this border region was 22.6 \pm 5.7 (s.d.) with an SNR of $17.0 \pm 2.5$ (s.d.). Excess peptide that was not rinsed away also collected non-specifically in the lumens of crypts of both polyps and normal mucosa, aiding in identification of the crypt distribution.

Figure 6 shows the receiver operating characteristic curve (ROC) for adenoma versus normal calculated using mean fluorescence signal values for three independent areas within each of 5 images of polyp and adjacent normal mucosa for each patient. Fluorescence intensity for differentiating between adenoma and normal mucosa yielded an optimum sensitivity of $81 \%$ and specificity of $78 \%$ for a contrast threshold of 4.94 , with p-value $<0.01$ using a two-sided student t-test.

In contrast to dysplastic polyps, fluorescence associated with hyperplastic polyps $(n=5)$ was predominantly associated with residual peptide collecting in regions between crypts and in crypt lumens. Hyperplastic crypts displayed slit-like or stellate-shaped lumens. Fluorescence from an additional $n=4$ endoscopically-polypoid lesions was similar to normal mucosa. These specimens were subsequently confirmed as containing no significant abnormality upon pathology examination.

Imaging using a scrambled control peptide ("QLMRPPV") showed no enhanced binding for either tubular adenomas $(n=5)$ or hyperplastic polyps $(n=2)$ as compared to normal mucosa. Imaging of hyperplastic polyps were similar to adenomatous polyps for scrambled control peptide. Fluorescence of the scrambled peptide localized to regions between crypts and in crypt lumens.

In addition to increased fluorescence due to peptide binding to colonocytes, signals were intermittently seen radiating from the lumens of crypts. Fluorescence was also occasionally seen in round structures within the colonocytes themselves. Rinsing with water for several seconds prior to imaging was required in order to remove excess peptide which may contribute to non-specific fluorescence. In addition, imaging performed after approximately $10 \mathrm{~min}$ (due to difficulty positioning the probe or patient peristalsis) yielded significantly decreased fluorescence signal. Photobleaching was observed during the imaging procedure on rare occasions when the probe was held stationary on the tissue surface for more than 5 seconds. No toxicity associated with peptide administration was observed in any patients.

A video showing real-time imaging of the border between a dysplastic polyp and normal mucosa is provided in Supplementary Video 1. A selection of images from adenomatous polyps from different patients is provided in Supplementary Figure 1 and a comparison of confocal fluorescence images showing peptide binding relative to autofluorescence background is provided in Supplementary Figure 2. Supplementary Figure 3 illustrates 
example confocal images of ex vivo peptide binding to excised tubular adenoma and normal tissue compared to scrambled control peptide.

\section{DISCUSSION}

Novel endoscopic imaging techniques such as optical coherence tomography (OCT) ${ }^{29-31}$, autofluorescence imaging (AFI) $)^{32-34}$, and narrow-band imaging (NBI) ${ }^{35-37}$ have been explored for the detection of dysplasia in the gastrointestinal tract. The use fluorescent imaging in combination with 5-aminolevulinic acid (5-ALA) $)^{38}$ as well as fluorescentlylabeled monoclonal antibodies against anticarcinoembryonic antigen has also been investigated ${ }^{39,40}$. The combination of targeted peptide probes and visualization using confocal microendoscopy offers a new method for facilitating in vivo optical imaging for early cancer detection, monitoring, and risk stratification. In our study, panning was performed using fresh tissue in order to identify peptides that bind to mucosal surface markers of colonic dysplasia. These experiments used fresh tissue as the target and were performed by clearing using dissected biopsies of normal human mucosa and panning using intact colon polyps. The selected peptide that was identified after three rounds of panning, "VRPMPLQ", was synthesized and conjugated with fluorescein for use in vivo as the means of validation. Imaging performed using a flexible confocal microendoscope demonstrated up to 50x increased fluorescence due to peptide binding to dysplastic polyps over normal mucosa.

In vivo imaging was performed at a $50 \mu \mathrm{m}$ working distance and with a $20 \mu \mathrm{m}$ axial resolution. Therefore we were able to predominantly image through the surface layer and visualize peptide binding to structures which were interpretable as crypt colonocytes. In these studies some accumulation of peptides were visible collecting in crypt lumens and between crypts after the washing procedure. Passive accumulation of peptide in the crypts is unavoidable, thus focusing analysis on specific regions enhanced identification of dysplasia. The process of applying and washing specific probes is one that is expected to improve as the technique is employed in more patients. Dysplasia-modified mucins may also be a viable target for peptide reagents. A number of both membrane-bound and secreted mucins are also known to be differentially expressed in colon adenocarcinomas, which may serve as targets given the presence of mucin in our intact polyp panning specimens ${ }^{41,42}$. In addition, it is possible that the absence of a particular protein or structure by dysplastic tissue may actually enhance binding to previously inaccessible sites. Alterations of tight junction structures in neoplasia serve as an example ${ }^{43,44}$. Therefore reagents discovered through intact tissue panning may have the advantage of selecting for targets that are not differentially expressed, but are clinically relevant because of accessibility. The molecular target of the 'VRPMPLQ' peptide is currently unknown and the sequence does not show full homology to any ligands for known receptors. This peptide does have partial homology (6 of 7 amino acids, ' $\mathbf{V} /$ LRPMPLQ') to the laminin-G domain of contactin-associated protein 1 (Caspr-1), which is present in human colon and small intestine and is thought to be involved in contactinmediated cell signaling ${ }^{45}$. One study has shown that contactin specific RNAi resulted in loss of metastatic and invasive capacity in in vivo models of lung adenocarcinoma, and it has been associated with clinical metastasis and patient survival ${ }^{46}$. Laminin-G domains are important for cell-cell interaction and therefore this homology suggests the target of the peptide may be surface-associated. It is expected that several peptides, each targeted towards a different molecular marker, may be required to optimize sensitivity and specificity for detecting dysplasia. Additional biopsy-screened phage are therefore being characterized. Finally, the selected peptide in this study was conjugated with fluorescein to the $\mathrm{N}$-terminus to simplify conjugation chemistry. Because the peptide insert is expressed with the Nterminus free on the phage during panning, $\mathrm{C}$-terminus conjugation with the addition of spacer residues may yield improved binding affinity and specificity. 
Panning using freshly resected tissue enabled identification of peptides preferentially selective for dysplastic relative to normal and hyperplastic mucosa. In vivo studies demonstrate that at least one of these peptides shows selective binding to dysplastic tissue over normal mucosa. While the performance of the current peptide is modest, refinement of the selection protocol to include more selection rounds as well as application in combination with other candidate peptides may result in improved sensitivity and specificity for binding to dysplasia. This peptide currently does not improve upon the reported performance of endoscopic confocal microscopy using intravenously administered fluorescein ${ }^{23}$. However, intravenously administered agents are less practical for clinical use as a screening tool. Peptides as well as other molecular probes also have the potential to provide quantitative analysis whereas non-specific contrast agents rely entirely on subjective interpretation of architectural morphology. The use of a targeted contrast agent for screening would ideally involve an instrument capable of wide-area surveillance. Currently available prototype fluorescence endoscopes for measuring bulk tissue fluorescence are low in resolution and tend to wash out the contrast from peptide binding because of a fairly significant amount of autofluorescence background. The approach of confocal microendoscopy in this study enabled validation of peptide binding based on visualization of microscopic features such as crypt shape in combination with colonocyte fluorescence. Future wide-area fluorescence imaging using targeted peptide reagents could be performed by modifying currently available prototype fluorescence endoscopic systems to excite and collect at longer wavelengths. The microstructural information provided by confocal microendoscopy and real-time imaging capability of this system provides a powerful adjunct to macroscopic imaging, enabling validation of local binding characteristics. Identification of dysplasiatargeting peptides may significantly enhance efforts to detect dysplastic lesions in patients with inflammatory bowel diseases or Barrett's esophagus, lesions for which the in vivo assessment of risk is currently not possible. This methodology can also be used to detect other epithelial cancers, assess disease margins during interventional procedures, monitor biomarkers for progression and/or recurrence of disease, and enable targeted drug delivery.

\section{METHODS \\ Cell Culture}

All cells were obtained from the American Type Culture Collection (ATCC, Manassas, VA). Hs738.st/int non-malignant human intestinal cells were maintained in Dulbecco's modified Eagle's medium (DMEM) supplemented with $4 \mathrm{mM}$ L-Alanyl-Glutamine with 4.5 $\mathrm{g} / \mathrm{L}$ D-glucose, $110 \mathrm{mg} / \mathrm{L}$ sodium pyruvate, $10 \%$ fetal bovine serum (FBS), 100 units $/ \mathrm{ml}$ penicillin, and $100 \mu \mathrm{g} / \mathrm{ml}$ streptomycin. HT29 human colorectal adenocarcinoma cells were maintained in McCoy's 5a medium (modified) supplemented with $1.5 \mathrm{mM} \mathrm{L-glutamine,}$ $10 \%$ FBS, 100 units $/ \mathrm{ml}$ penicillin, and $100 \mu \mathrm{g} / \mathrm{ml}$ streptomycin. All cells were grown at $37^{\circ}$ $\mathrm{C}$ in 5\% $\mathrm{CO}_{2}$, fed fresh media every 3-4 days, and passaged using trypsin EDTA $0.05 \%$ upon reaching confluence.

\section{Generation of Cleared Phage Library}

A total of $1.3 \times 10^{11}$ plaque forming units (pfu) of the PhD7 phage library (New England Biolabs, Beverly, MA), consisting of $\sim 50$ copies of $2.8 \times 10^{9}$ peptide 7 -mers fused to a minor coat protein (pIII) of M13 phage, was incubated in $16.5 \mathrm{~mL}$ of complete media with confluent HS738 cells in a $75 \mathrm{~cm}^{2}$ flask. Cells were incubated at $37^{\circ} \mathrm{C}$ for 70 minutes (min) with gentle agitation. The supernatant containing unbound phage was collected, precipitated with PEG-NaCl, resuspended in complete media, and incubated for another round with confluent HS738 cells for 2 hours (h). Samples of the supernatant were collected every 1015 minutes in order to monitor the unbound phage titer and determine when steady-state clearing was reached. The remaining unbound phage was collected, precipitated with PEG- 
$\mathrm{NaCl}$, and resuspended in $1 \mathrm{~mL}$ of phosphate-buffered saline (PBS) for panning with fresh biopsy tissue.

\section{Patient Study and Tissue Specimen Collection}

IRB approval was granted by Stanford University Medical Center and the VA Palo Alto Health Care Systems. Patients undergoing routine screening colonoscopy were recruited and informed consent was obtained. Subjects with coagulation abnormalities, poor preparation for colonoscopy, or low tolerance for colonoscopy were excluded from the study. For the ex vivo portion of the study, tissue specimens were collected from pinch biopsies of polyp and adjacent normal mucosa obtained during conventional endoscopic examination. Tissue biopanning was performed using these biopsies within $15 \mathrm{~min}$ of tissue excision. For the in vivo portion of the study, a complete blood count (CBC), platelets, and chemistries (Panel-20), including blood urea nitrogen (BUN), creatinine (Cr), glucose (Glu), and liver function tests (LFTs), were performed in order to assess for potential peptide toxicity. These labs were performed prior to the procedure and follow-up lab tests were performed approximately 24 hours after the procedure. Abnormal tests were repeated until the value normalized. The fluorescein-conjugated VRPMPLQ peptide and the scrambled peptide was topically administered in 20 patients and 6 patients, respectively, prior to imaging. Patients ranged in age from 40 to 83 years old (mean $63 \pm 10$ ). A total of 24 adenomatous polyps and 7 hyperplastic polyps were imaged.

\section{Panning with Excised Tissue}

The first round of selection was performed using a cleared library where non-specifically bound phage was removed using HS738 cells. Amplification was not performed between selection rounds to avoid the possibility of introducing a screening bias. To compensate for low phage recovery in the absence of amplification, panning was restricted to 3 rounds, yielding a reasonably large titer of clones which could be assessed using binding assays. Enzymatic tissue dissociation was also avoided in order to prevent possible receptor alteration during tissue digestion. Clearing was performed prior to each panning step in order to reduce non-specifically binding phage. This procedure was chosen to bias selection towards markers present only on dysplastic mucosa rather than on those that are overexpressed, with the intention of optimizing fluorescence signal-to-background during endoscopic imaging. All tissues used for the panning were stained using hematoxylin and eosin $(\mathrm{H} \& \mathrm{E})$ and read by two independent pathologists. A schematic of the panning protocol is shown in Figure 1.

Round 1-Approximately $4.25 \times 10^{8}$ plaque-forming units (pfu) of the cleared library was suspended in $500 \mu \mathrm{L}$ of DMEM in a sterile $1.5 \mathrm{~mL}$ Eppendorf tube. One biopsy of a colon polyp and one biopsy of adjacent normal mucosa was obtained from each patient and rinsed in $500 \mu \mathrm{L}$ of fresh DMEM. Normal mucosa was dissociated using two scalpel blades and incubated with the precleared library for $15 \mathrm{~min}$ with gentle agitation at room temperature to preabsorb phage binding to normal colon mucosa. Contents were then centrifuged for $5 \mathrm{~min}$ at $6000 \mathrm{rpm}$ and supernatant containing unbound phage transferred to a fresh Eppendorf tube. The pellet consisting of homogenized normal mucosa was resuspended in $100 \mu \mathrm{L}$ of PBS, centrifuged again, and the supernatant containing residual unbound phage was added to the first supernatant. Unbound phage were then incubated at room temperature with the intact colon polyp for 15 min with gentle agitation. The polyp was transferred to a fresh Eppendorf and the bound phage were eluted with $400 \mu \mathrm{L}$ of glycine $\mathrm{HCl}(\mathrm{pH} 2.2)$ for 5 min. The polyp was transferred into a fresh Eppendorf tube and the remaining bound phage solution was neutralized with $80 \mu \mathrm{L}$ of Tris- $\mathrm{HCl}(\mathrm{pH} 9.1)$. The polyp was rinsed with 100 $\mu \mathrm{L}$ of PBS and this rinse was added to the Eppendorf containing the eluted phage. The polyp was then fixed in $10 \%$ buffered formalin and submitted for routine histology. Each 
supernatant following panning with a polyp was retained pending the results of pathology so that either the bound or unbound phage could be retained depending on the pathology of the samples.

Round 2-The eluted phage from the polyp from the first round (verified to be tubular adenoma) was split into three Eppendorf tubes each containing $\sim 200 \mu \mathrm{L}$ of biopsy-eluted phage. Three biopsies of colon polyp and adjacent normal mucosa were obtained from 3 different patients. Each tube was separately incubated with homogenized normal mucosa and panned with intact polyp as described in Round 1. Polyps were formalin fixed and submitted for routine histology and pathology evaluation.

Round 3-Pathology results from Round 2 indicated the polyps were hyperplastic (one polyp), inflamed mucosa (second polyp), or contained only a small region of focal adenomatous change (third polyp). Therefore panning in Round 2 was considered a clearing round and the panning supernatant from all three polyps were combined into one Eppendorf tube. This tube was vortexed and split again into three Eppendorf tubes, each containing $\sim 200 \mu \mathrm{L}$ of cleared phage from Round 2 . One tube was incubated with homogenized normal mucosa and panned with intact polyp as described in Round 1. The two remaining tubes were panned only with polyps. Both polyp-eluted phage and polyp panning supernatants were retained pending results of pathology.

\section{Cell-binding Assays}

Of the total $\sim 6000$ clones eluted from adenomas in Round 3 of selection, 40 were randomly selected and amplified for initial testing using HT29 cells. Imaging was initially performed on 4 pools of 10 phage clones and one pool was split into 10 individual clones for further testing. HT29 cells were grown to confluence in 8-well chamber slides and incubated for $1 \mathrm{~h}$ at $37^{\circ} \mathrm{C}$ with pools of biopsy-eluted phage at $2 \times 10^{10}$ pfu total concentration in $300 \mu \mathrm{L}$ complete media. Cells were then rinsed once with PBS, blocked using $1 \%$ bovine serum albumin (BSA) for $5 \mathrm{~min}$, rinsed again with PBS, and incubated with $300 \mu \mathrm{L}$ of 1:500 mouse M13 monoclonal antibody conjugated with FITC (Fitzgerald Industries, Concord, MA) for $1 \mathrm{~h}$ at $37^{\circ} \mathrm{C}$. Following antibody incubation, cells were rinsed twice with PBS and imaging was performed using a commercial fluorescence confocal microscope (Leica TCS SP2) at 10x through 63x magnification. Increased fluorescence intensity was used as an indicator of phage binding to select the most promising phage clone for further assays.

Preferential binding to HT29 cells over HS738 cells was assessed by determining the titer of bound phage. The most promising phage clone from the previous assay plus 10 additional randomly selected clones from Round 3 of panning were amplified. Cells were grown to confluence in 24-well plates, rinsed once with PBS, and incubated for $15 \mathrm{~min}$ at $37^{\circ} \mathrm{C}$ with phage at $10^{10} \mathrm{pfu}$ total concentration in $300 \mu \mathrm{L}$ complete media with $0.5 \%$ BSA. After incubation, cells were washed four times with $300 \mu \mathrm{L}$ of PBS plus $0.5 \%$ BSA and $0.05 \%$ Tween 20. Bound phage were subsequently eluted using $400 \mu \mathrm{l}$ of glycine $\mathrm{HCl}$ for $3 \mathrm{~min}$, removed, and neutralized using $80 \mu \mathrm{L}$ of Tris- $\mathrm{HCl}$ prior to titering. Binding of phage to HT29 cells over HS738 cells was assessed relative to wild-type insertless phage used as a control. Titering assays were performed in quadruplicate in order to obtain mean and standard deviation values.

\section{Ex Vivo Imaging of Phage Binding}

Imaging of binding of biopsy-eluted phage to human tissue specimens obtained using the protocol described above was performed within $2 \mathrm{~h}$ of excision. Imaging was initially performed on 4 pools of 10 phage clones and one pool exhibiting increased fluorescence intensity was split and 6 individual clones for further testing. Fresh biopsies of normal 
mucosa and colon polyps were rinsed in $500 \mu \mathrm{L}$ of fresh DMEM, then incubated for $15 \mathrm{~min}$ at room temperature with $5 \times 10^{9}$ pfu phage in $300 \mu \mathrm{L}$ DMEM. Biopsies were then rinsed twice with PBS, blocked using 1\% BSA for 5 min, rinsed again with PBS, and incubated with $300 \mu \mathrm{L}$ of 1:1000 mouse M13 monoclonal antibody conjugated with FITC for $1 \mathrm{~h}$ at room temperature. Following antibody incubation, biopsies were rinsed twice with PBS and imaging was performed using a commercial confocal microscope (BioRad MRC-1024) at 10x through 60x magnification. Biopsies were subsequently formalin-fixed, H\&E-stained, and read by staff pathologists at the Palo Alto VA hospital.

\section{Synthesis of Peptide Conjugates}

The peptide sequence 'VRPMPLQ' identified by phage display was conjugated at the Nterminus to fluorescein via an aminohexanoic acid linker. Fluorescein was chosen because of its prior regulatory approval and compatibility with the confocal microendoscope. The scrambled peptide sequence 'QLMRPPV' was synthesized also linked to fluorescein for use as a control. All peptides were synthesized using standard (F)luorenyl-(m)eth(o)xy(c)arbonyl (FMOC) chemistry, purified to minimum $98 \%$ purity using high-performance liquid chromatography (HPLC), and analyzed by reverse phase HPLC and mass spectrometry.

\section{Patient Study}

Imaging was first performed prior to peptide administration to assess for detectable autofluorescence. Approximately $3-6 \mathrm{~mL}$ of peptide at a concentration of $100 \mu \mathrm{M}$ was then administered topically to approximately $1-2 \mathrm{~cm}^{2}$ of the surface of the colon using a standard endoscopic spray catheter. Excess peptide was removed by gently rinsing the region with water. Imaging was performed within $\sim 5$ min of peptide administration using the Cellvizio®-GI confocal fluorescence imaging system (Mauna Kea Technologies, Paris, France). The excitation wavelength used for imaging was $488 \mathrm{~nm}$ and fluorescence was collected between 505-800 $\mathrm{nm}$. The fibered confocal microscope was passed through the instrument channel of a standard medical colonoscope (Olympus CFQ-160). Fluorescence videos were collected in vivo at 12 frames per second up to depths of approximately $50 \mu \mathrm{m}$ beneath the mucosal surface with a resolution of $2.5 \mu \mathrm{m}$ (transverse) and $20 \mu \mathrm{m}$ (axial). Imaging of the polyp and adjacent endoscopically-appearing normal mucosa was performed before and after peptide administration. No other dyes were used for confocal microscopy. Mean imaging time was approximately 10 minutes for each polyp and fluorescence videos were coregistered with endoscopic video to record the imaging location. After imaging, the polyp was removed according to standard protocol, submitted for routine histology, and read by staff pathologists at the Palo Alto VA hospital.

\section{Ex Vivo Peptide Imaging}

Human tissue specimens obtained using the protocol described above were collected and rinsed briefly in PBS. Intact biopsies of polyp and normal mucosa were separated in three pieces. Two pieces were incubated in either $100 \mu \mathrm{M}$ 'VRPMPLQ' peptide or control 'QLMRPPV' scrambled peptide for $~ 10$ minutes followed by rinsing for 5 minutes in PBS. Peptide-treated tissue was subsequently immediately frozen in OCT and cut the same day into 7 um sections. Sections were allowed to dry overnight, rinsed for $5 \mathrm{~min}$ in PBS to remove OCT, fixed in methanol for 5 minutes, and mounted in anti-fade medium containing DAPI (Vector Laboratories, Burlingame, CA). Sections were imaged using a commercial confocal microscope (Leica SP2 AOBS) at 40x magnification. The third piece was formalinfixed, H\&E-stained, and read by staff pathologists at the Palo Alto VA hospital. 


\section{Image and Statistical Analysis}

For each polyp and normal region imaged in vivo, 5 representative images were selected based on the following criteria: 1) minimum motion artifact, 2) lack of stool or excess mucus preventing contact with the mucosa, and 3) recognizable crypt structure in the images. Fluorescence signal intensity was calculated by taking the mean of a pixel array corresponding to a $25 \times 25 \mu \mathrm{m}$ square region within the colonocyte layer for three sites within each image. The signal noise was calculated by taking the standard deviation of the values in this pixel array. The fluorescence contrast was then obtained from the ratio of the mean polyp to the mean normal fluorescence intensity for each patient. The signal-to-noise (SNR) was obtained from the ratio of the mean polyp signal to signal noise for each image site within each patient. Average fluorescence signal contrast and SNR attributed to the peptide was then calculated by averaging the values obtained for each patient. Standard errors of the mean (s.e.m.) were reported based on $n=19$ polyps. The receiver operating characteristic (ROC) for adenoma versus normal was calculated using mean fluorescence signal values for each image site using a total of $n=285$ values for adenoma and $n=285$ values for normal. All images were interpreted using corresponding H\&E stained histology for comparison.

\section{Supplementary Material}

Refer to Web version on PubMed Central for supplementary material.

\section{Acknowledgments}

The authors acknowledge funding support from the National Institutes of Health, including U54 CA105296 (NCI), K08 DK067618 (NIDDK), P30 DK56339 (DDC Pilot Award), Doris Duke Charitable Foundation, the Stanford School of Medicine Dean's Fellowship and the John A. and Cynthia Fry Gunn Research Fund. We thank Dr. Jon Kosek his support with histopathological interpretation and Mauna Kea Technologies for technical support and use of the Cellvizio®-GI imaging system.

\section{References}

1. Vogelstein B, et al. Genetic alterations during colorectal-tumor development. N Engl J Med. 1988; 319:525-32. [PubMed: 2841597]

2. Winawer SJ, et al. Prevention of colorectal cancer by colonoscopic polypectomy. The National Polyp Study Workgroup. N Engl J Med. 1993; 329:1977-81. [PubMed: 8247072]

3. American Cancer Society. Cancer Facts \& Figures. 2006.

4. Fujii T, Rembacken BJ, Dixon MF, Yoshida S, Axon AT. Flat adenomas in the United Kingdom: are treatable cancers being missed? Endoscopy. 1998; 30:437-43. [PubMed: 9693889]

5. Hart AR, Kudo S, Mackay EH, Mayberry JF, Atkin WS. Flat adenomas exist in asymptomatic people: important implications for colorectal cancer screening programmes. Gut. 1998; 43:229-31. [PubMed: 10189849]

6. Kudo S, et al. Colonoscopic diagnosis and management of nonpolypoid early colorectal cancer. World J Surg. 2000; 24:1081-90. [PubMed: 11036286]

7. Jaramillo E, et al. Small, flat colorectal neoplasias in long-standing ulcerative colitis detected by high-resolution electronic video endoscopy. Gastrointest Endosc. 1996; 44:15-22. [PubMed: 8836711]

8. Mayer R, Wong WD, Rothenberger DA, Goldberg SM, Madoff RD. Colorectal cancer in inflammatory bowel disease: a continuing problem. Dis Colon Rectum. 1999; 42:343-7. [PubMed: 10223754]

9. Garcea G, et al. Molecular biomarkers of colorectal carcinogenesis and their role in surveillance and early intervention. Eur J Cancer. 2003; 39:1041-52. [PubMed: 12736102]

10. Goldsmith SJ. Receptor imaging: competitive or complementary to antibody imaging? Semin Nucl Med. 1997; 27:85-93. [PubMed: 9144853] 
11. Scott JK, Smith GP. Searching for peptide ligands with an epitope library. Science. 1990; 249:38690. [PubMed: 1696028]

12. Kelly KA, Jones DA. Isolation of a colon tumor specific binding peptide using phage display selection. Neoplasia. 2003; 5:437-44. [PubMed: 14670181]

13. Kelly K, Alencar H, Funovics M, Mahmood U, Weissleder R. Detection of invasive colon cancer using a novel, targeted, library-derived fluorescent peptide. Cancer Res. 2004; 64:6247-51. [PubMed: 15342411]

14. Zangani D, et al. Multiple differentiation pathways of rat mammary stromal cells in vitro: acquisition of a fibroblast, adipocyte or endothelial phenotype is dependent on hormonal and extracellular matrix stimulation. Differentiation. 1999; 64:91-101. [PubMed: 10234806]

15. Walters RW, et al. Basolateral localization of fiber receptors limits adenovirus infection from the apical surface of airway epithelia. J Biol Chem. 1999; 274:10219-26. [PubMed: 10187807]

16. Pasqualini R, Ruoslahti E. Organ targeting in vivo using phage display peptide libraries. Nature. 1996; 380:364-6. [PubMed: 8598934]

17. Rajotte $\mathrm{D}$, et al. Molecular heterogeneity of the vascular endothelium revealed by in vivo phage display. J Clin Invest. 1998; 102:430-7. [PubMed: 9664085]

18. Carson-Walter EB, et al. Cell surface tumor endothelial markers are conserved in mice and humans. Cancer Res. 2001; 61:6649-55. [PubMed: 11559528]

19. Arap W, et al. Steps toward mapping the human vasculature by phage display. Nat Med. 2002; 8:121-7. [PubMed: 11821895]

20. Krag DN, et al. Selection of tumor-binding ligands in cancer patients with phage display libraries. Cancer Res. 2006; 66:7724-33. [PubMed: 16885375]

21. Maruta F, et al. Use of a phage display library to identify oligopeptides binding to the lumenal surface of polarized endothelium by ex vivo perfusion of human umbilical veins. J Drug Target. 2003; 11:53-9. [PubMed: 12852441]

22. Hoffman JA, et al. Progressive vascular changes in a transgenic mouse model of squamous cell carcinoma. Cancer Cell. 2003; 4:383-91. [PubMed: 14667505]

23. Kiesslich R, et al. Confocal laser endoscopy for diagnosing intraepithelial neoplasias and colorectal cancer in vivo. Gastroenterology. 2004; 127:706-13. [PubMed: 15362025]

24. Polglase AL, et al. A fluorescence confocal endomicroscope for in vivo microscopy of the upperand the lower-GI tract. Gastrointest Endosc. 2005; 62:686-95. [PubMed: 16246680]

25. Laemmel E, et al. Fibered confocal fluorescence microscopy (Cell-viZio) facilitates extended imaging in the field of microcirculation. A comparison with intravital microscopy. Journal of Vascular Research. 2004; 41:400-11. [PubMed: 15467299]

26. Thiberville $\mathrm{L}$, et al. In vivo imaging of the bronchial wall microstructure using fibered confocal fluorescence microscopy. Am J Respir Crit Care Med. 2007; 175:22-31. [PubMed: 17023733]

27. Meining A, Bajbouj M, Schmid RM. Confocal fluorescence microscopy for detection of gastric angiodysplasia. Endoscopy. (in press).

28. Meining A, Schwendy S. In-vivo histopathology of lymphocytic colitis. Gastrointestinal Endoscopy. (in press).

29. Jackle S, et al. In vivo endoscopic optical coherence tomography of the human gastrointestinal tract--toward optical biopsy. Endoscopy. 2000; 32:743-9. [PubMed: 11068832]

30. Sivak MV Jr, et al. Gastrointest Endosc. 2000; 51:474-9. [PubMed: 10744825]

31. Pfau PR, et al. Criteria for the diagnosis of dysplasia by endoscopic optical coherence tomography. Gastrointest Endosc. 2003; 58:196-202. [PubMed: 12872085]

32. Wang TD, et al. Fluorescence endoscopic imaging of human colonic adenomas. Gastroenterology. 1996; 111:1182-91. [PubMed: 8898631]

33. Brand S, et al. Detection of colonic dysplasia by light-induced fluorescence endoscopy: a pilot study. Int J Colorectal Dis. 1999; 14:63-8. [PubMed: 10207734]

34. Haringsma J, et al. Autofluorescence endoscopy: feasibility of detection of GI neoplasms unapparent to white light endoscopy with an evolving technology. Gastrointest Endosc. 2001; 53:642-50. [PubMed: 11323596] 
35. Gono K, et al. Appearance of enhanced tissue features in narrow-band endoscopic imaging. $\mathbf{J}$ Biomed Opt. 2004; 9:568-77. [PubMed: 15189095]

36. Machida $\mathrm{H}$, et al. Narrow-band imaging in the diagnosis of colorectal mucosal lesions: a pilot study. Endoscopy. 2004; 36:1094-8. [PubMed: 15578301]

37. Su MY, et al. Comparative study of conventional colonoscopy, chromoendoscopy, and narrowband imaging systems in differential diagnosis of neoplastic and nonneoplastic colonic polyps. Am J Gastroenterol. 2006; 101:2711-6. [PubMed: 17227517]

38. Messmann H, Knuchel R, Baumler W, Holstege A, Scholmerich J. Endoscopic fluorescence detection of dysplasia in patients with Barrett's esophagus, ulcerative colitis, or adenomatous polyps after 5-aminolevulinic acid-induced protoporphyrin IX sensitization. Gastrointest Endosc. 1999; 49:97-101. [PubMed: 9869731]

39. Folli S, et al. Immunophotodiagnosis of colon carcinomas in patients injected with fluoresceinated chimeric antibodies against carcinoembryonic antigen. Proc Natl Acad Sci U S A. 1992; 89:79737. [PubMed: 1518823]

40. Keller R, Winde G, Terpe HJ, Foerster EC, Domschke W. Fluorescence endoscopy using a fluorescein-labeled monoclonal antibody against carcinoembryonic antigen in patients with colorectal carcinoma and adenoma. Endoscopy. 2002; 34:801-7. [PubMed: 12244502]

41. Ho SB, et al. Heterogeneity of mucin gene expression in normal and neoplastic tissues. Cancer Res. 1993; 53:641-51. [PubMed: 7678777]

42. Gendler SJ, Spicer AP. Epithelial mucin genes. Annu Rev Physiol. 1995; 57:607-34. [PubMed: 7778880]

43. Martinez-Palomo A. Ultrastructural modifications of intercellular junctions between tumor cells. In Vitro. 1970; 6:15-20. [PubMed: 4331913]

44. Soler AP, et al. Increased tight junctional permeability is associated with the development of colon cancer. Carcinogenesis. 1999; 20:1425-31. [PubMed: 10426787]

45. Peles E, et al. Identification of a novel contactin-associated transmembrane receptor with multiple domains implicated in protein-protein interactions. Embo J. 1997; 16:978-88. [PubMed: 9118959]

46. Su JL, et al. Knockdown of contactin-1 expression suppresses invasion and metastasis of lung adenocarcinoma. Cancer Res. 2006; 66:2553-61. [PubMed: 16510572] 


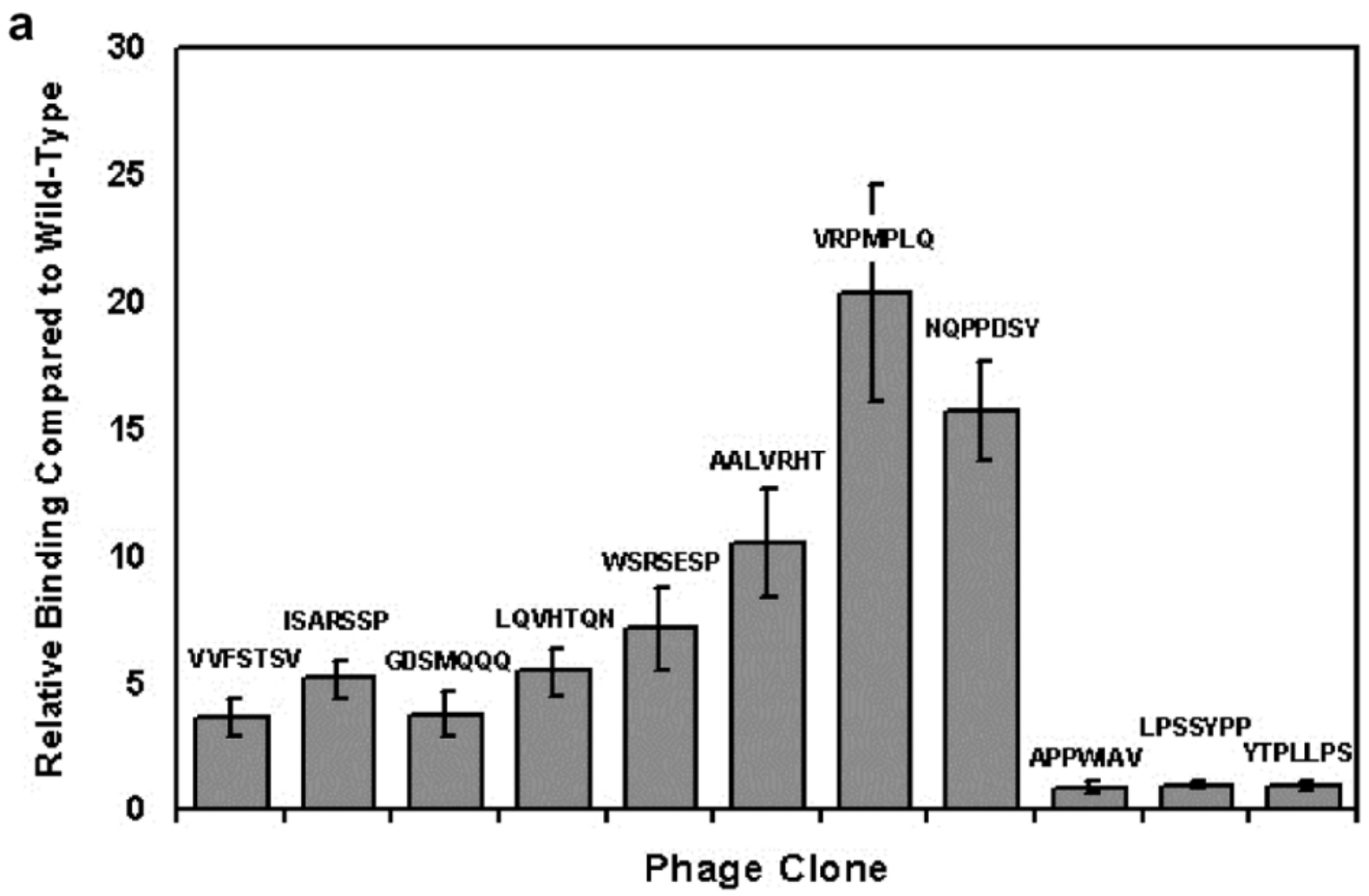



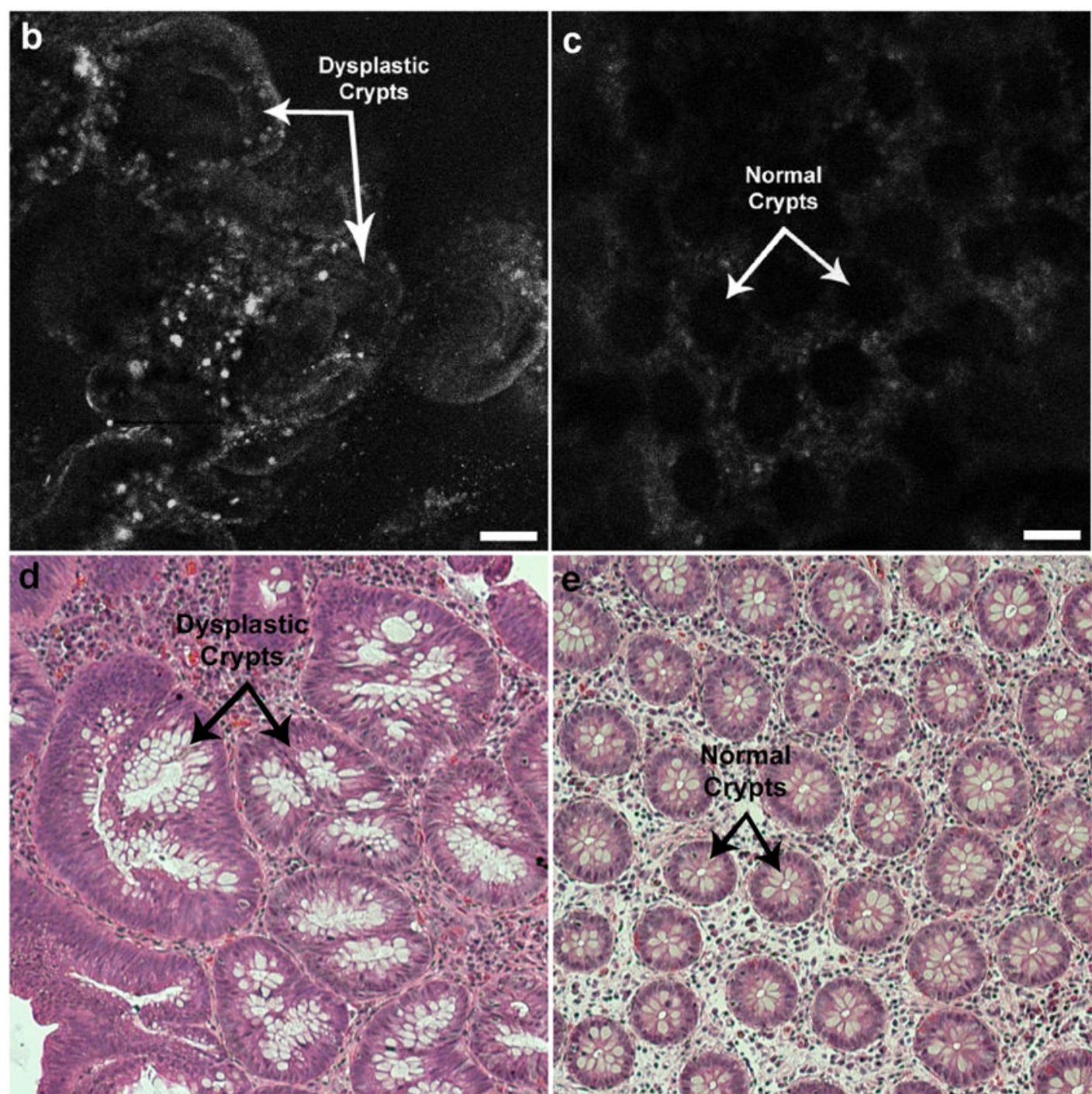

Figure 1.

Schematic of peptide reagent development procedure. An M13 phage library was first cleared with non-malignant epithelial cells and freshly excised normal human colonic mucosa. Panning was then performed using freshly resected human colon polyps. Peptides displayed by phage that bound to dysplastic tissue were characterized, synthesized, and conjugated to fluorescein for use as imaging reagents during endoscopy. 

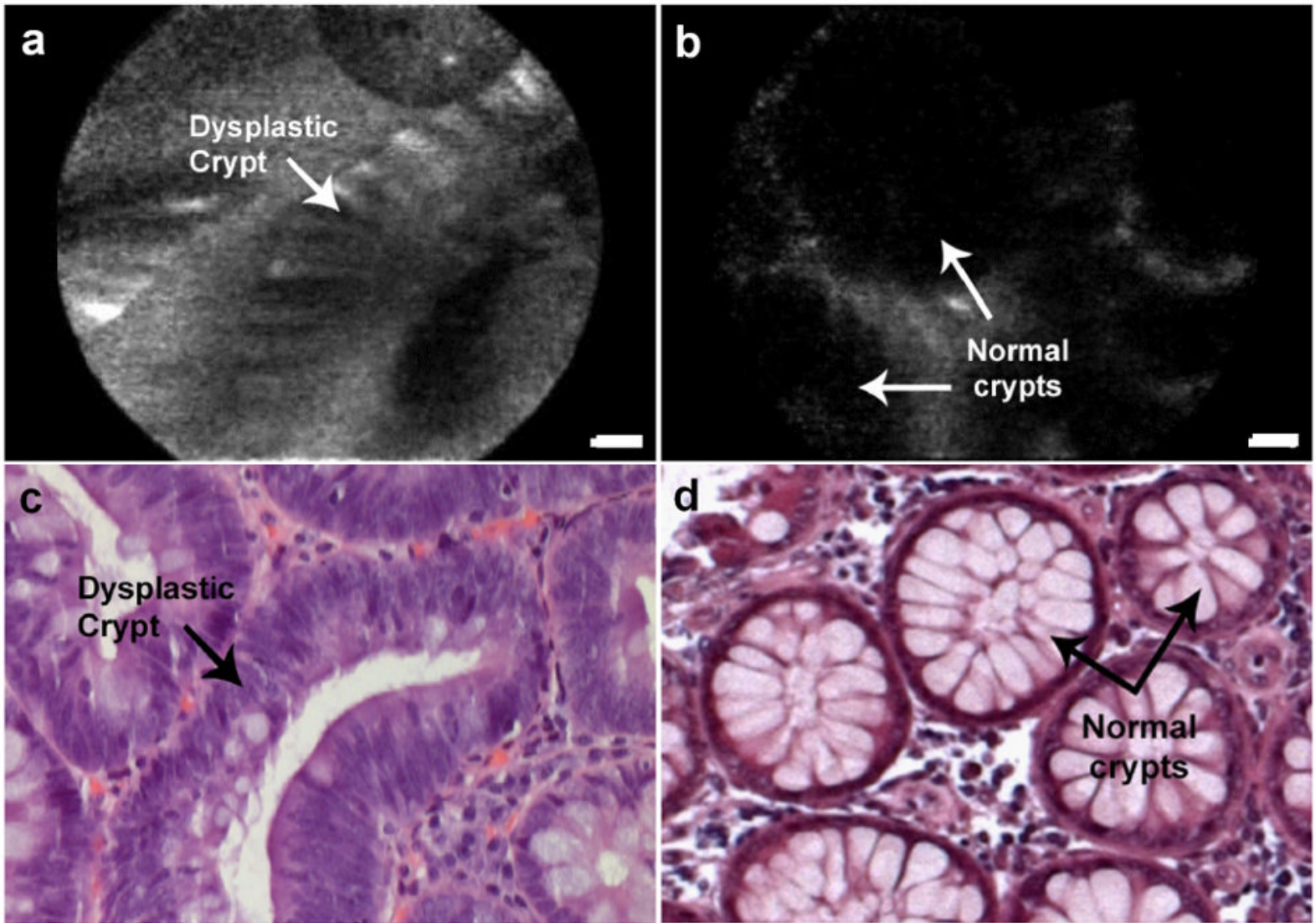

Figure 2.

Cell binding assay comparing clone 'VRPMPLQ' against other candidate clones eluted from Round 3 of selection. Data shows relative binding to HT29 (transformed) cells over HS738 (non-transformed) cells, normalized to wild-type insertless M13 phage (binding =1). Error bars reflect standard error of the mean (s.e.m.) for $\mathrm{n}=4$ experiments. 

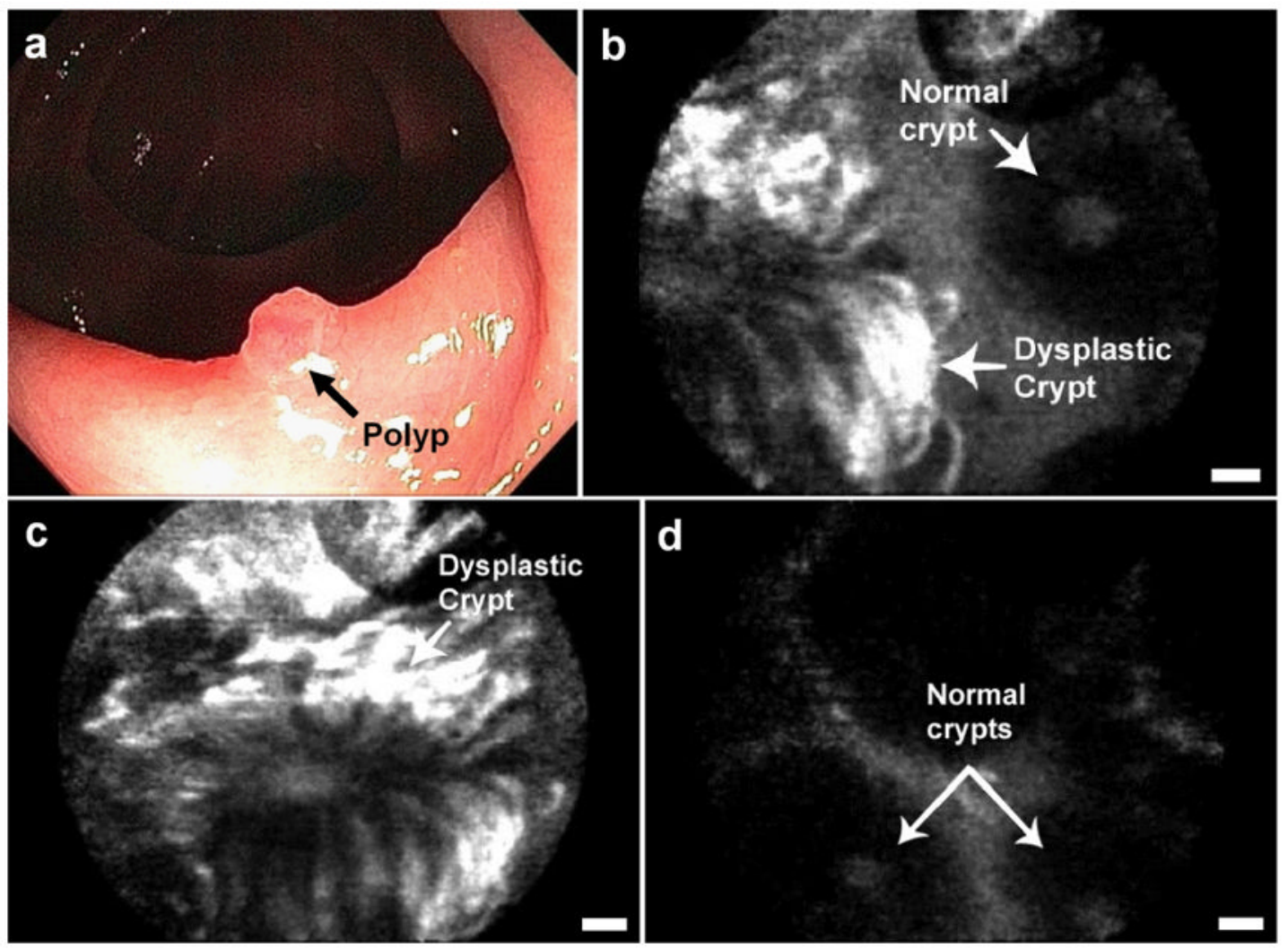

Figure 3.

Confocal images of phage binding to (a) freshly excised colon adenoma, (b) normal mucosa. Specimens were incubated with $5 \times 10^{9}$ pfu phage bearing peptide 'VRPMPLQ.' Presence of phage is detected through FITC-conjugated M13 monoclonal antibody. (c, d): Histology, H\&E, 4x. Scale bars: $50 \mu \mathrm{m}$. 


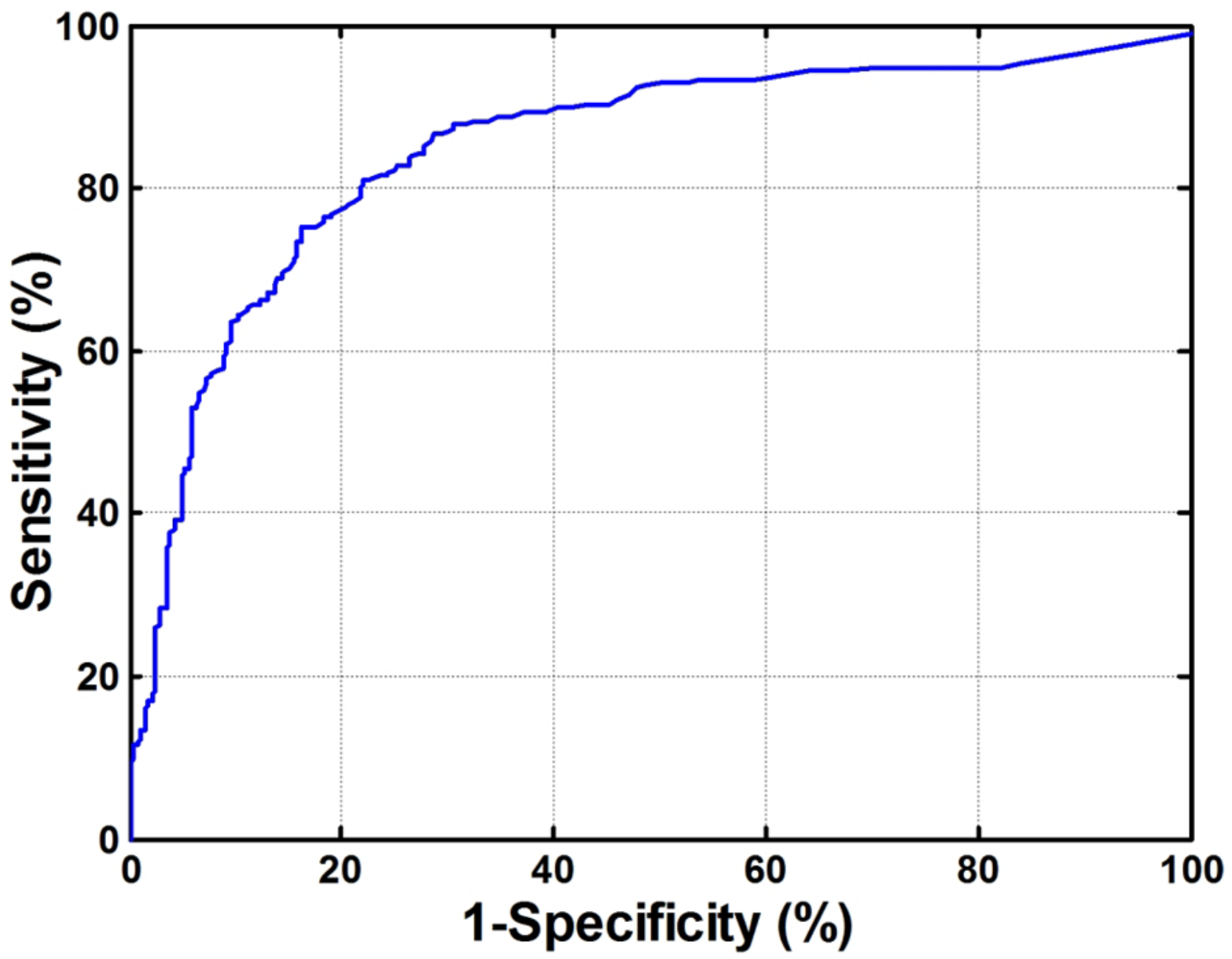

Figure 4.

In vivo confocal fluorescence images of peptide binding to (a) dysplastic colon polyp, (b) adjacent normal mucosa. (c, d): Histology, H\&E, 20x. Scale bars: $20 \mu \mathrm{m}$. 


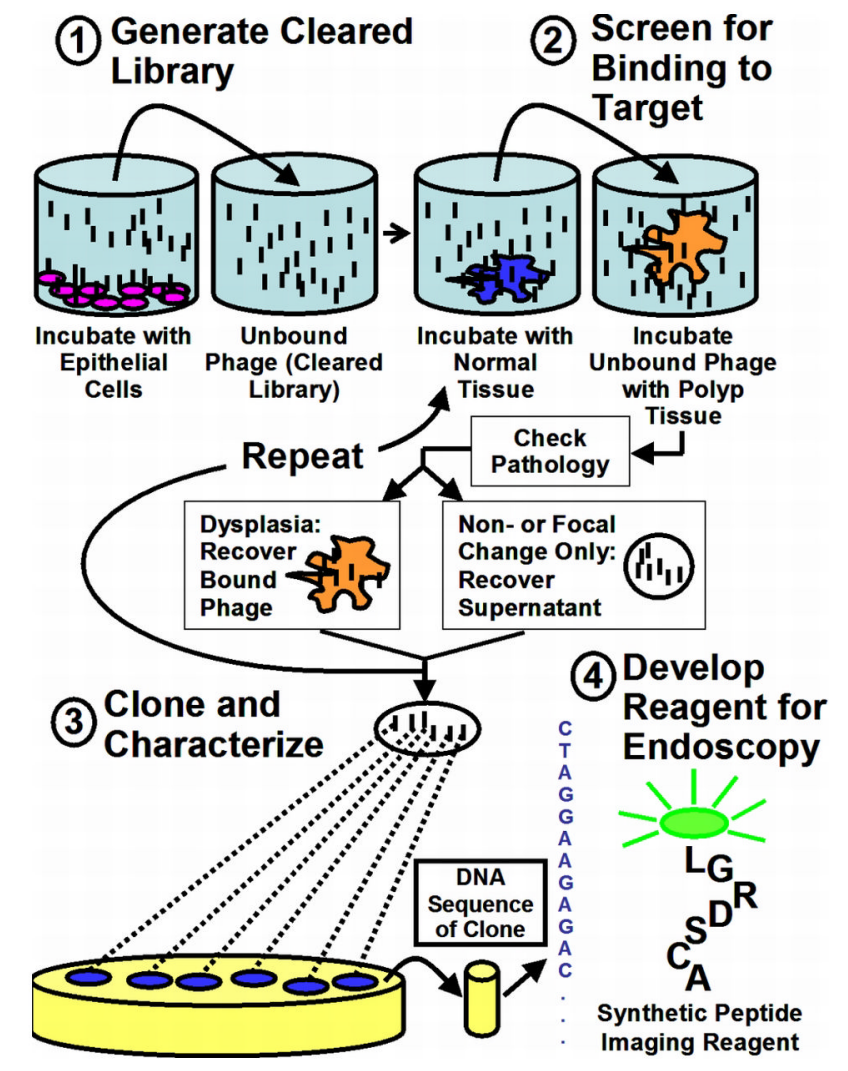

Figure 5.

In vivo confocal fluorescence images of the border between colonic adenoma and normal mucosa showing peptide binding to dysplastic colonocytes: (a) Endoscopic view. (b) Border. (c) Dysplastic crypt. (d) Adjacent mucosa. Scale bars: $20 \mu \mathrm{m}$. 